\title{
Effect of Mach Number on Airfoil Characteristics at Reynolds Number of 3,000*
}

\author{
Seiichiro Morizawa, ${ }^{1 \dagger}$ Taku Nonomura, ${ }^{2)}$ Akira Oyama, ${ }^{2)}$ Kozo FuJII, ${ }^{2)}$ and Shigeru OBAYASHI ${ }^{1)}$ \\ ${ }^{1)}$ Institute of Fluid Science, Tohoku University, Sendai, Miyagi 980-8577, Japan \\ ${ }^{2)}$ Institute of Space and Astronautical Science, Japan Aerospace Exploration Agency, Sagamihara, Kanagawa 252-5210, Japan
}

The effects of Mach number at $R e=3,000$ for different airfoils (NACA0012, NACA0002, NACA4412, NACA4402) with thickness and camber geometries are investigated for the propeller blade design of a Mars airplane. The present study shows that thin and cambered airfoils have larger variations in $C_{l}$ than symmetric airfoils. As for thin airfoils, $C_{l}$ at higher $\alpha$ has rapid increases when the $M_{\infty}$ is low. This is because the flow separation occurs at the leading edge, and the flow is reattached on the airfoil surface. However, the rapid increase in $C_{l}$ disappear as $M_{\infty}$ increases because the flow reattachment does not occurs. As for cambered airfoils, the decrease in $C_{l}$ becomes larger than that on the symmetric airfoils when $M_{\infty}$ is higher. This is because $C_{p}$ near the leading edge on the lower surface is smaller than that on the upper surface and the high-speed region on the lower side of the leading edge is enlarged as $M_{\infty}$ increases. Then, the $M_{c r}$ at $R e=3,000$ tends to be larger than that predicted by linear theory.

Key Words: Low-Reynolds Number, Mach-number Effect, Airfoil Characteristics, Mars Airplane

\section{Nomenclature}

$c$ : chord length of airfoil

$C_{d}$ : drag coefficient

$C_{f}$ : local skin-friction coefficient

$C_{p}$ : surface pressure coefficient

$C_{l}$ : lift coefficient

$\operatorname{div} \vec{V}$ : divergence of velocity

$l / d$ : lift-to-drag ratio

$M_{\infty}$ : freestream Mach number

$M_{c r}$ : critical Mach number

$r$ : radius-direction position of propeller blade

$R$ : radius-length of propeller blade

$R e$ : Reynolds number based on the chord length and freestream velocity

$u$ : chord-length-direction velocity

$U_{\infty}$ : freestream velocity

$x$ : chord-length-direction coordinate of airfoil

$\alpha$ : angle of attack

$\gamma$. specific heat ratio

$\omega_{y}$ : spanwise vorticity

\section{Introduction}

Many studies have measured aerodynamic characteristics of standard airfoils. Vast quantities of data are available covering a wide range of Reynolds and Mach numbers for a variety of natural and manmade flying objects (for example, see Ref. 1)). These data can be categorized into three types,

C 2018 The Japan Society for Aeronautical and Space Sciences

*Presented at the 46th Fluid Dynamics Conference/32nd Aerospace

Numerical Simulation Symposium, 3-4 July 2014, Hirosaki, Japan.

Received 17 November 2017; final revision received 13 May 2018; accepted for publication 15 June 2018.

†Corresponding author, morizawa@mech.tottori-u.ac.jp as shown in Fig. 1,2) i.e., low-speed data, high-speed data, and ultrahigh altitude aircraft data.

Many airplanes capable of flying in the Martian atmosphere have been proposed. ${ }^{3-5)}$ One technical difficulty comes from the flight condition on Mars. First, the Martian atmosphere is very thin and has roughly one-hundredth the density of Earth's atmosphere. Then, the Reynolds number $(R e)$ for the flight is very low. Second, because the air temperature on Mars is lower than that on Earth and the Martian atmosphere is comprised mainly of carbon dioxide, the Mach number for the flight is roughly 1.5 times higher than that on Earth. For successful flight under these atmospheric conditions, the effects of the Mach number on airfoil characteristics during the low $R e$ flight conditions must be considered. Japanese researchers have studied the feasibility of a Mars exploration mission using a propeller-driven Mars airplane. ${ }^{6}$ Kojima et al., ${ }^{7)}$ Anyoji et al., ${ }^{8)}$ and Lee et al. ${ }^{9}{ }^{9}$ studied the aerodynamics of the main wing of this Mars airplane under the flight conditions it would likely encounter (Re ranging from 10,000 to 100,000). The remaining issue is the aerodynamics of the propeller blade for the propulsion system.

According to the blade element theory, ${ }^{10)}$ the efficiency of a propeller blade can improve when the lift-to-drag ratio of its airfoil at each section is relatively high. However, the characteristics of the propeller blade are not adequately understood because the flight condition of the propeller blade for the Mars airplane is low Reynolds number and high Mach number regimes. Thus, the tip speed of the propeller blade reaches the high-subsonic regime and/or the higher speed region to produce sufficient thrust while $R e$ based on the airfoil-chord length is very low $(1,000$ to 10,000$)$. As illustrated in Fig. 1, such low Reynolds number and high Mach number flow conditions are not covered in the existing airfoil aerodynamics database. 


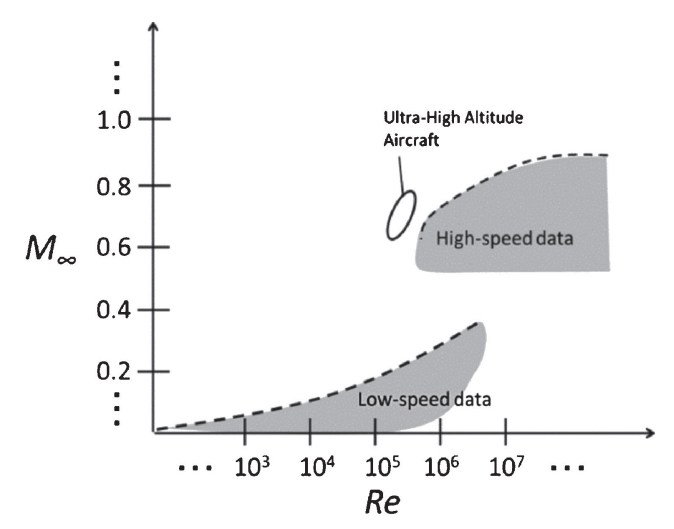

Fig. 1. Available airfoil data plotted in terms of chord Re and flight Mach number. ${ }^{2)}$

In 2006, Takaki ${ }^{11)}$ investigated Mach number's effects on the aerodynamic characteristics of the NACA4402 airfoil at $R e=1,000$ using a Reynolds-averaged Navier-Stokes simulation and showed that the lift-to-drag ratio decreases by approximately $10 \%$ as the Mach number increases from 0.1 to 0.7. In 2012, Suwa et al. ${ }^{12)}$ tested a flat plate and a triangular airfoil in the Mars wind tunnel at Tohoku University in Japan. The Reynolds number was 3,000 or 10,000 , and the Mach number was $0.15,0.5$, or 0.7 . They found that the maximum lift-to-drag ratio decreases as the Mach number increases. These findings are a helpful guide for the present purpose, but data about different airfoil geometries of thickness and camber with the Mach number effect and the related flow phenomena are also needed to improve the efficiency of a propeller blade to realize the Mars airplane.

Therefore, the objective of this study is to investigate the aerodynamic performance of different airfoil geometries of thickness and camber with effects of the Mach number at $R e$ $=3,000$, which is corresponding to the cruising flight of the propeller blade for the Mars airplane. ${ }^{13)}$ Then, the related flow phenomena were discussed. For these purpose, the aerodynamic performances at $R e=3,000$ with the Mach number effects are evaluated for the NACA0012, NACA0002, NACA4412, and NACA4402 airfoils. Concerning to flow phenomena, the difference of the critical Mach number at $R e=3,000$ is investigated by comparing numerical simulations of the NACA0012 airfoil at $R e=3,000$ and the predictions of a linear theory. Then, the relationship between the separation point and the flow field was also investigated. In addition, the flow fields of thin airfoils are evaluated by the NACA0002 and NACA4402 airfoils, and the flow fields of cambered airfoils are also evaluated by the NACA4412 and NACA4402 airfoils.

\section{Problem Setting}

Table 1 represents the information of a Mars-airplanepropeller blade at the cruising flight condition, and Fig. 2 shows the shape of a propeller blade for this airplane. ${ }^{13)}$ Based on Table 1 and Fig. 2, the variations of $R e$ and $M_{\infty}$ against normalized radial-direction are determined as shown
Table 1. Information of a Mars-airplane-propeller blade at cruising flight condition.

\begin{tabular}{lc}
\hline Cruising speed of Mars airplane $[\mathrm{m} / \mathrm{s}]$ & 50 \\
Total thrust force of propellers $[\mathrm{N}]$ & 2.2 \\
Number of propellers [N] & 2 \\
A thrust force a propeller blade [N] & 1.1 \\
Number of propeller blades & 4 \\
Radial length of propeller blades [m] & 0.25 \\
Revolving speed of propeller blade [rps] & 80 \\
Propeller efficiency & 0.606 \\
Torque [m.s] & 0.179 \\
\hline
\end{tabular}

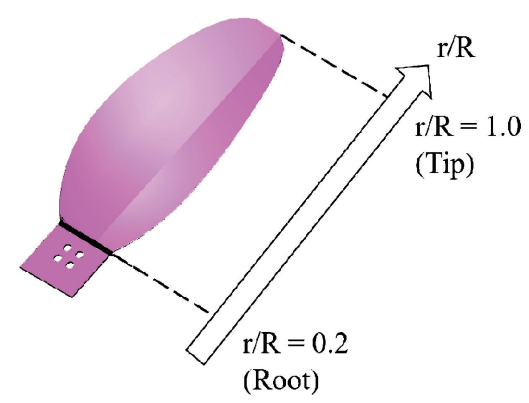

Fig. 2. Shape of a propeller blade for the Mars airplane.

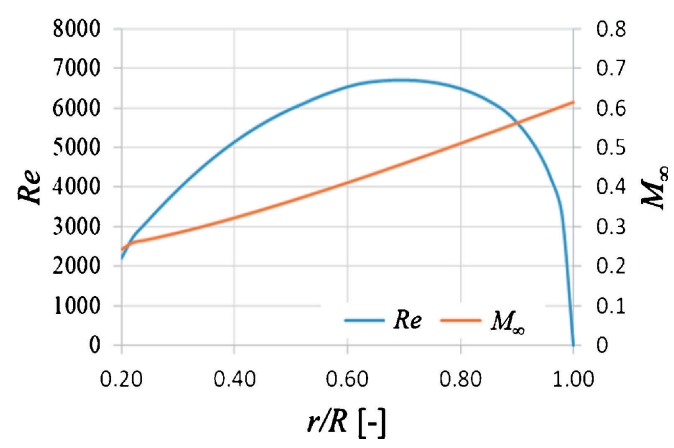

Fig. 3. Spanwise variations of $R e$ and $M_{\infty}$ of a propeller blade at the cruising flight of the Mars airplane.

in Fig. 3. As shown in Fig. 3, the flow condition near at root and tip positions of the propeller blade is approximately $R e=3,000$. In addition, Fig. 3 shows that the flow condition near at tip positions is relatively higher Mach number. Thus, it is important to consider the effect of the Mach number at $R e=3,000$ when designing the propeller blade.

The effects of the Mach number at the low Reynolds number condition on the aerodynamic characteristics were simulated for the NACA0012, NACA0002, NACA4412, and NACA4402 airfoils, as shown in Fig. 4. The Reynolds number is 3,000, and the Mach number varies from 0.2 to 0.9 in increments of 0.1 . The angle of attack changes from $0^{\circ}$ to $9^{\circ}$ in increments of $1^{\circ}$. Thus, the simulations were performed at eight Mach numbers and 10 angles of attack for a total of 80 simulations, making 320 cases altogether. The viscosity of the air, the speed of sound, the specific heat ratio, and the Prandtl number are $1.8 \times 10^{-5} \mathrm{~Pa} \cdot \mathrm{s}, 343.6 \mathrm{~m} / \mathrm{s}, 1.4$, and 0.72 , respectively. Note that, with regard to these above values, the condition of the atmospheric property is corresponding to Earth. Discussion on the effects of the Mach number in 


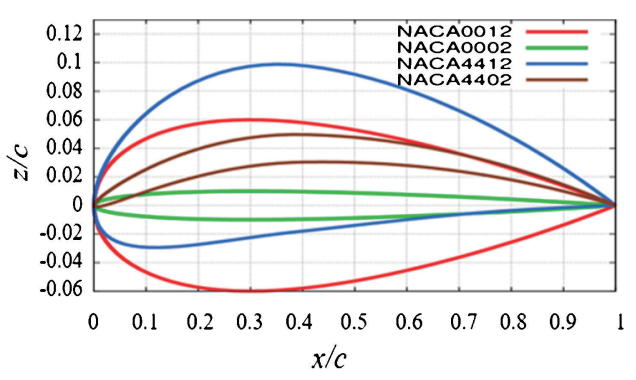

Fig. 4. Comparison of selected airfoils.

this study was conducted based on the similarity rule of fluid dynamics.

\section{Computational Method}

The Navier-Stokes solver LANS3D ${ }^{14-16)}$ was used to solve compressible Navier-Stokes equations generalized in curvilinear coordinates. The flow fields are assumed to be unsteady two-dimensional laminar flow over the entire region because the corresponding $R e$ is 3,000, and the influence on three-dimensional flow field is small. To discretize these equations, we evaluated spatial derivatives of the convective terms using a simple high-resolution upwind scheme (SHUS $)^{17)}$ with the third-order monotone upstream scheme for the conservation laws (MUSCL). ${ }^{18)}$ Viscous terms are computed using the second-order central difference scheme. An alternate directional implicit symmetric Gauss-Seidel scheme (ADI-SGS) ${ }^{19,20)}$ for the second-order backward differencing with three subiterations is adopted for the time integration. The computational time steps are $1.6 \times 10^{-4}$ in nondimensional time, such that the maximum CourantFriendrichs-Levy number becomes approximately 1.6. Noslip flow conditions are applied to the airfoil surface. A C-type grid topology is adopted where the outer boundary layer of the grid is located at a distance 20 times the chord length away from the airfoil. Grid convergence study was checked using three different grids: $249 \times 51,497 \times 101$, and $615 \times 101$. The $497 \times 101$ grid is employed as the standard grid for this study. The minimum size of the grid in the normal direction is defined as $0.01 / \sqrt{R e}$.

For validation of our numerical approach, computational results are compared with experimental data for the NACA0012-34 airfoil at $R e=4,100$ and $M_{\infty}=0.22 .^{21)}$ Figure 5 compares the current numerical simulation and the experiment in terms of airfoil's lift and drag coefficients. This result indicates that the present simulation accurately predicts aerodynamic performances at angles of attack $9^{\circ}$ or less, although the lift and drag coefficients are overestimated at higher angles. This overestimation is expected because the flow separates from the leading edge of the airfoil for angles of attack above $9^{\circ}$ under the present condition. However, qualitative predictions of aerodynamic performance are still possible because the trends shown in Fig. 5 are well reproduced.

In addition, the flow fields around the NACA0012 airfoil at Reynolds numbers $1 \times 10^{4}, 3 \times 10^{4}, 5 \times 10^{4}$ and the pre-

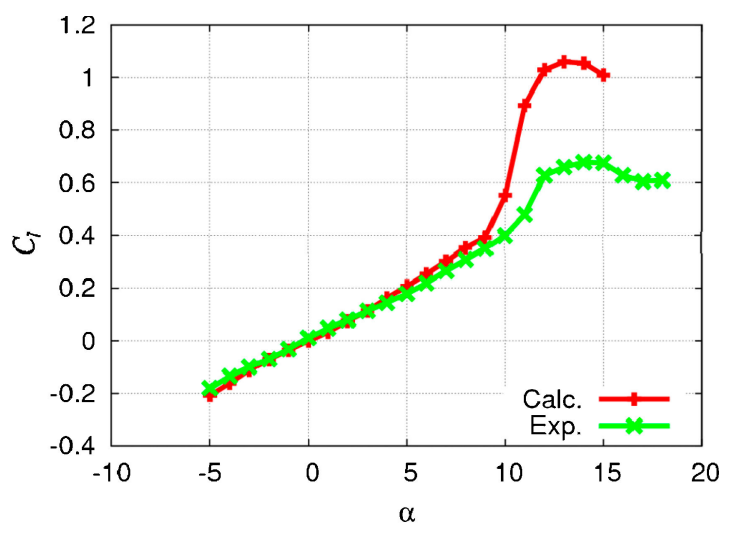

(a) Lift coefficient

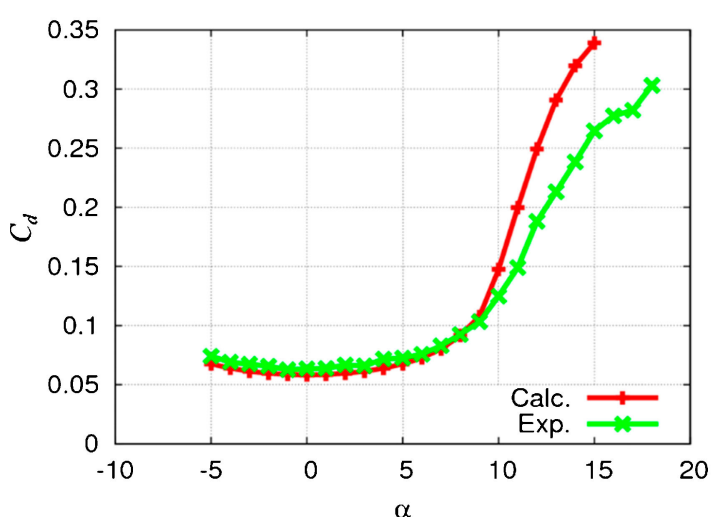

(b) Drag coefficient

Fig. 5. Comparison of aerodynamic performance between the current numerical simulation and the experiment.

dictability of the aerodynamic characteristics have been examined by our research group in a previous publication. ${ }^{22}$ ) That study showed that the laminar separation point of the flow can be predicted accurately. We conclude that the present approach can predict airfoil characteristics qualitatively, demonstrating its appropriateness for numerical simulations of flow fields at relatively low Reynolds numbers. The LANS3D solver has also been shown to reproduce shock-related phenomena (for example, see Refs.14)-16)) such as the flow around the wing. Thus, the implementation of current numerical simulations for this study can predict aerodynamic properties in higher Mach number conditions.

\section{Results and Discussion}

\subsection{Aerodynamic performance of different airfoil geom- etry with Mach number effects}

This section compares the aerodynamic performance of the four airfoils (NACA0012, NACA0002, NACA4412, and NACA4402) in the conditions simulated and discusses the influence of airfoil geometries, such as thickness and camber, on aerodynamic performance. The relations of airfoil geometries are summarized in Fig. 6.

Lift and drag coefficients, $C_{l}$ and $C_{d}$, at different Mach numbers are plotted against angles of attack for each of the four airfoils in Figs. 7 and 8. $C_{l}$ of the NACA0012 airfoil varies less than it does for other airfoils. Large variations 
in $C_{l}$ are observed when the geometry of the airfoil has thin and cambered. For thin airfoils, $C_{l}$ rapidly increases at high angles of attack when the Mach number is low. Thus, as indicated by the arrows in the figure, $C_{l}$ rapidly increases for the NACA0002 airfoil at $M_{\infty}=0.2, \alpha=6^{\circ}$ and at $M_{\infty}=$ $0.5, \alpha=9^{\circ}$. On the other hand, $C_{l}$ rapidly increases for the NACA4402 airfoil at $M_{\infty}=0.2, \alpha=6^{\circ}$ (also marked with an arrow in the lower right panel of the figure). These increase in $C_{l}$ disappear as the Mach number increases. At low angles of attack, the decreases in $C_{l}$ on the cambered airfoils becomes larger than that on the symmetric airfoils, and the negative value of $C_{l}$ on the NACA4412 airfoil is also observed when the Mach number is higher. In addition, increases in $C_{d}$ as the Mach number increases are confirmed to be an overall tendency of these airfoils.

Figure 9 plots the lift-to-drag ratio, $l / d$, at different Mach numbers for each airfoil. The decrease in $l / d$ of these airfoils

\begin{tabular}{|c|c|c|}
\hline & Symmetric & Cambered \\
\hline 䆃 & NACA0012 & NACA4412 \\
\hline 貝 & NACA0002 & NACA4402 \\
\hline
\end{tabular}

Fig. 6. Summary of selected airfoils. is observed as the Mach number increases, except for the thin airfoil at lower angles of attack with $M_{\infty}=0.2$. This is because, as mentioned above, $C_{d}$ increases and $C_{l}$ decreases when the Mach number is higher. However, as shown in Fig. 9, the decrease in $l / d$ of thin airfoils is more pronounced at high angles of attack when the Mach number is relatively high (in particular, NACA0002: $\alpha=6^{\circ}$ and NACA4402: $\alpha=9^{\circ}$ ). Comparison of Figs. 7-9 indicates that $l / d$ is affected by the drop in $C_{l}$. The decrease of $l / d$ observed with the cambered airfoils as the Mach number increases is larger than the corresponding decrease of $l / d$ of symmetric airfoils. This result indicates that $l / d$ was affected by the drop in $C_{l}$. These geometric effects on aerodynamic performance and flow phenomena at different Mach numbers are discussed in more detail below.

\subsection{Flow phenomena of airfoils with Mach number effects}

\subsubsection{Fundamental characteristics of the NACA0012 airfoil}

The flow condition at $R e=3,000$ has a dominant influence of the viscosity. It is important to compare the influence of the viscosity at $R e=3,000$ with the Mach number effect to high-speed data of Fig. 1. For this purpose, the results of the NACA0012 airfoil between the Navier-Stokes simulations at $R e=3,000$ and the linearized potential theory (the Prandtl-Glauert rule) are compared. The linear theory for the NACA0012 airfoil is mentioned in a reference. ${ }^{23)}$ According to this reference, the linear theory could predict the critical Mach number of the NACA0012 airfoil at $\alpha=0^{\circ}$ within about one percent compared to the experimental

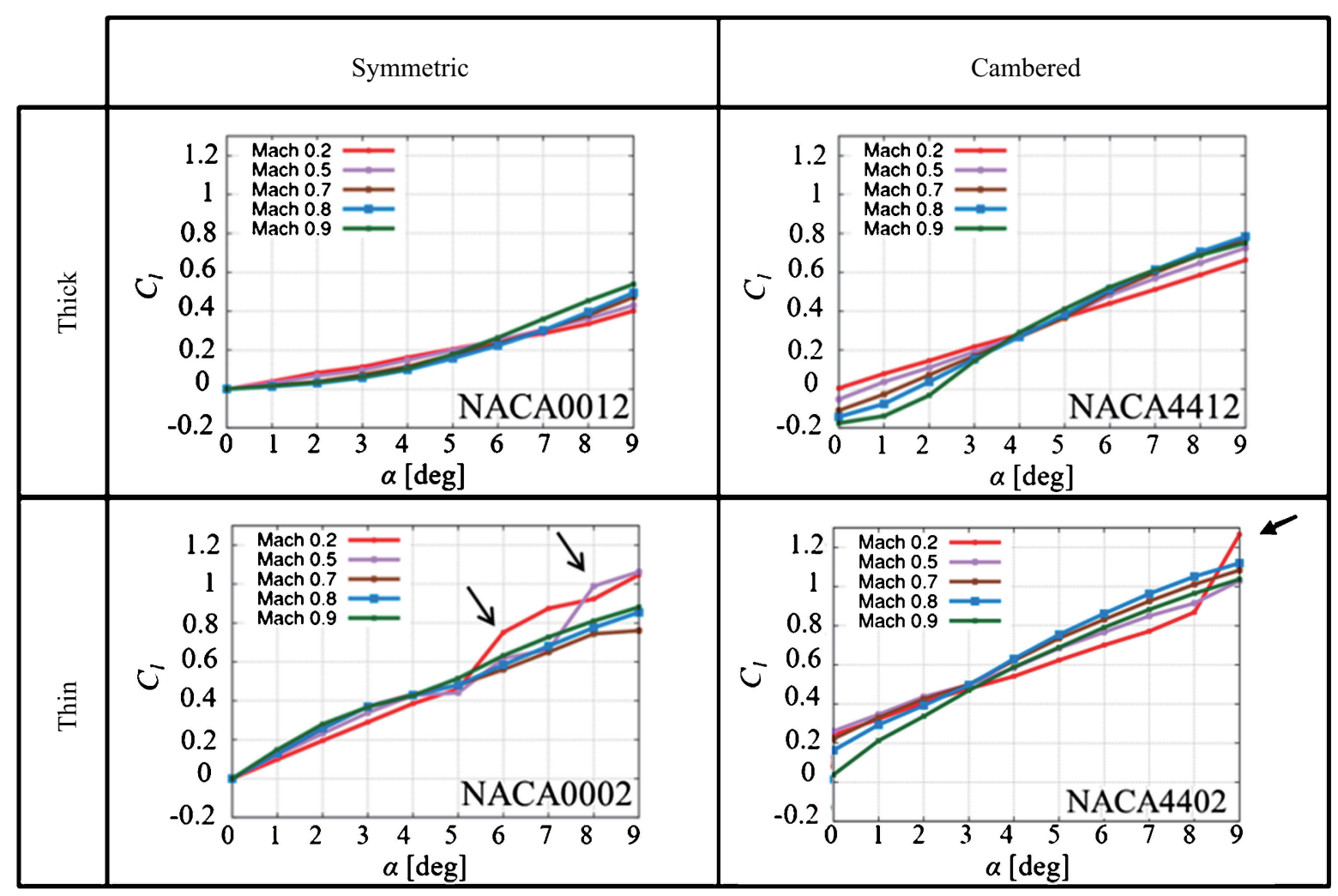

Fig. 7. Comparison of $C_{l}$ curves at different Mach numbers. 


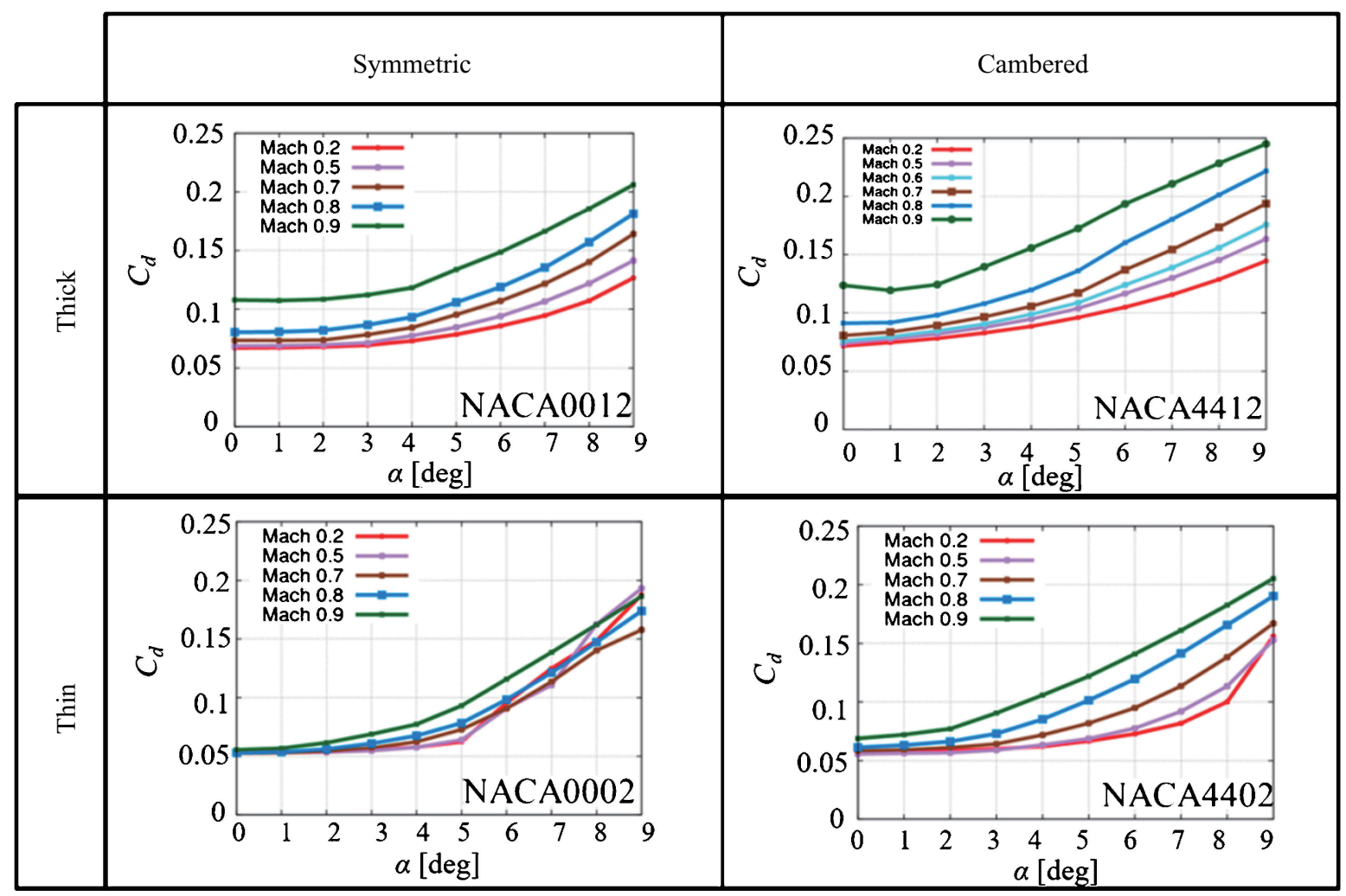

Fig. 8. Comparison of $C_{d}$ curves at different Mach numbers.

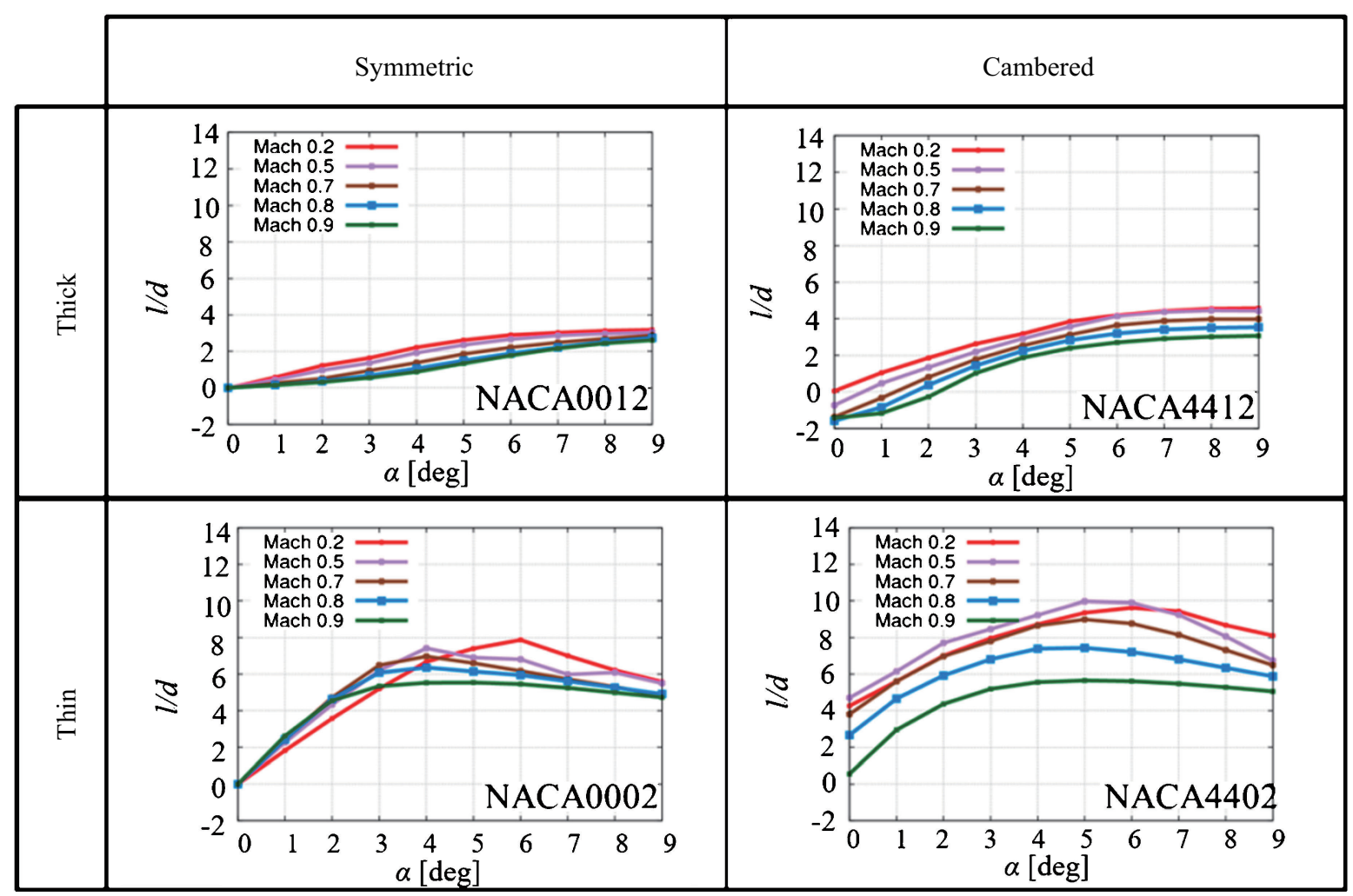

Fig. 9. Comparison of $l / d$ curves at different Mach numbers.

measurement at $R e=5.34 \times 10^{6}$. In this study, the results of the linear theory are derived from the results of numerical simulations at $M_{\infty}=0.2$ and $\alpha=0^{\circ}$.

The variation in the minimum value of $C_{p}$ with freestream
Mach number between the results of the numerical simulations and the linear theory is shown in Fig. 10. The value of $M_{c r}$ is also estimated in this figure. The value of $M_{c r}$ can be obtained at the intersection point of the variation in 


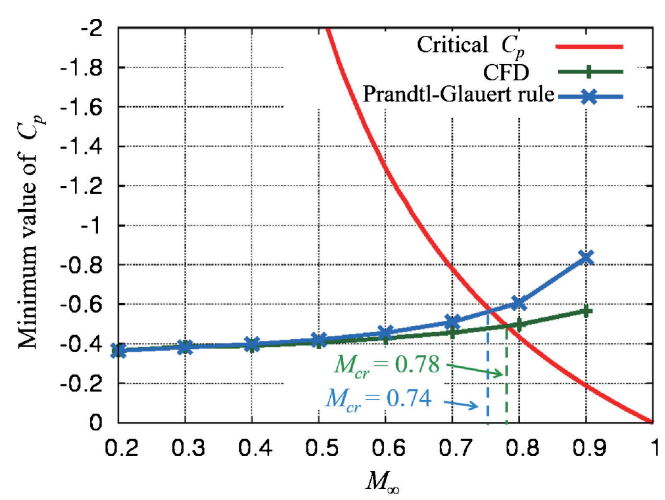

Fig. 10. Variation in the minimum value of $C_{p}$ with freestream Mach number between the results of the numerical simulation and the linear theory.

the minimum $C_{p}$ and the critical $C_{p}$ lines. The value of critical $C_{p}$ can be obtained as following equation.

Critical $C_{p}=\frac{2}{\gamma M_{\infty}^{2}}\left[\left(\frac{1+\{(\gamma-1) / 2\} M_{\infty}^{2}}{1+(\gamma-1) / 2}\right)^{\gamma /(\gamma-1)}-1\right]$

As shown in Fig. 10, the $C_{p}$ values from both the numerical simulations and the linear theory increase as the Mach number increases. However, large differences in $C_{p}$ are observed above $M_{\infty}=0.7$. The value of $M_{c r}$ from the linear theory is 0.74 and the value of $M_{c r}$ from numerical simulations is 0.78 , as shown in Fig. 10. Thus, the shock wave at $R e=3,000$ is generated at a higher Mach number in the numerical simulation than in the linear theory. This means that the magnitude of peak pressure near the leading edge on the upper surface is less in numerical simulations than in predicted the linear theory.

Figures 11 and 12 show the chordwise position of the separation point at $R e=3,000$ and the distribution of $\operatorname{div} \vec{V}$ at $\alpha=9^{\circ}$, respectively. The separation point is defined as the most upstream point with $C_{f}=0$ on the upper surface. ${ }^{24)}$ The location on the generations of the shock wave are observed by $\operatorname{div} \vec{V}=-1$. At low angles of attack (especially, at $\alpha=0^{\circ}$ ), the separation points move forward as the Mach number increases (see Fig. 11). This similar result is reported that the forward movement of the laminar separation point occurs because the variation of the momentum thickness depends only little on the Mach number, whereas the displacement thickness increases considerably as the Mach number is increased. ${ }^{25)}$ Meanwhile, at higher angles of attack (especially, at $\alpha=9^{\circ}$ ), the separation points at $M_{\infty}=0.5$ and 0.7 move slightly forward than that at $M_{\infty}=0.2$. These movements of the separation points at $\alpha=9^{\circ}$ is smaller than that at $\alpha=0^{\circ}$. Then, the separation points for $\alpha=9^{\circ}$ at $M_{\infty}=0.8$ and 0.9 move downstream because of the generations of the shock wave (see Figs. 11 and 12). In addition, the separation point at $M_{\infty}=0.9$ is located more on the trailing edge side than that at $M_{\infty}=0.8$. This result indicates that the supersonic region at $M_{\infty}=0.9$ becomes larger than that at $M_{\infty}=0.8$, and the generation of the shock wave

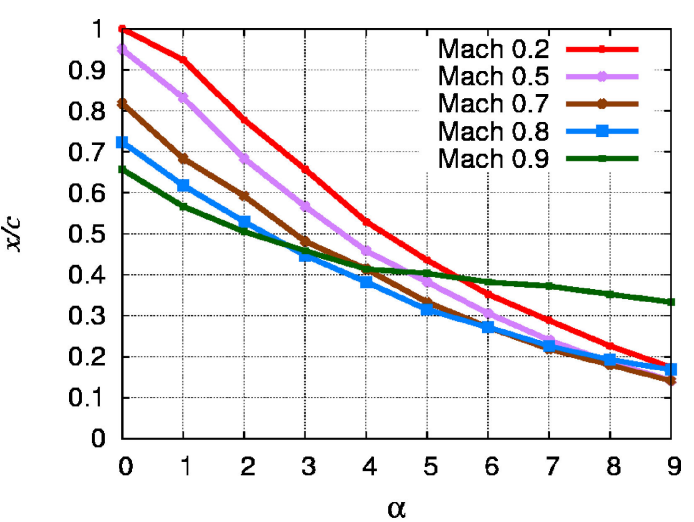

(a) Overview of all angles of attack

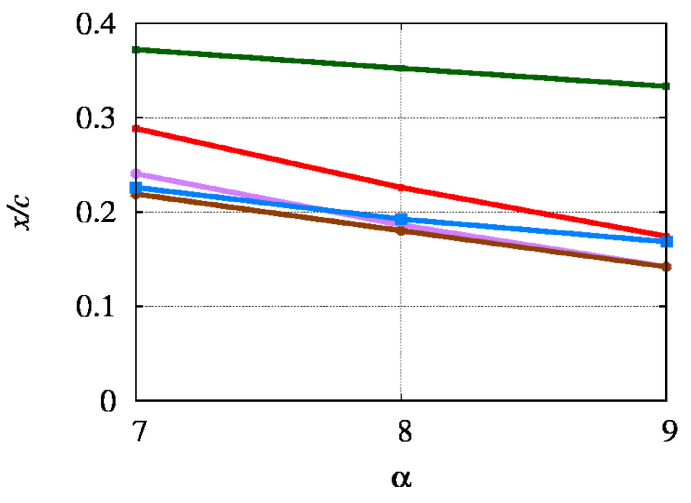

(b) Enlarge view at higher angles of attack

Fig. 11. Chordwise position of the separation point.

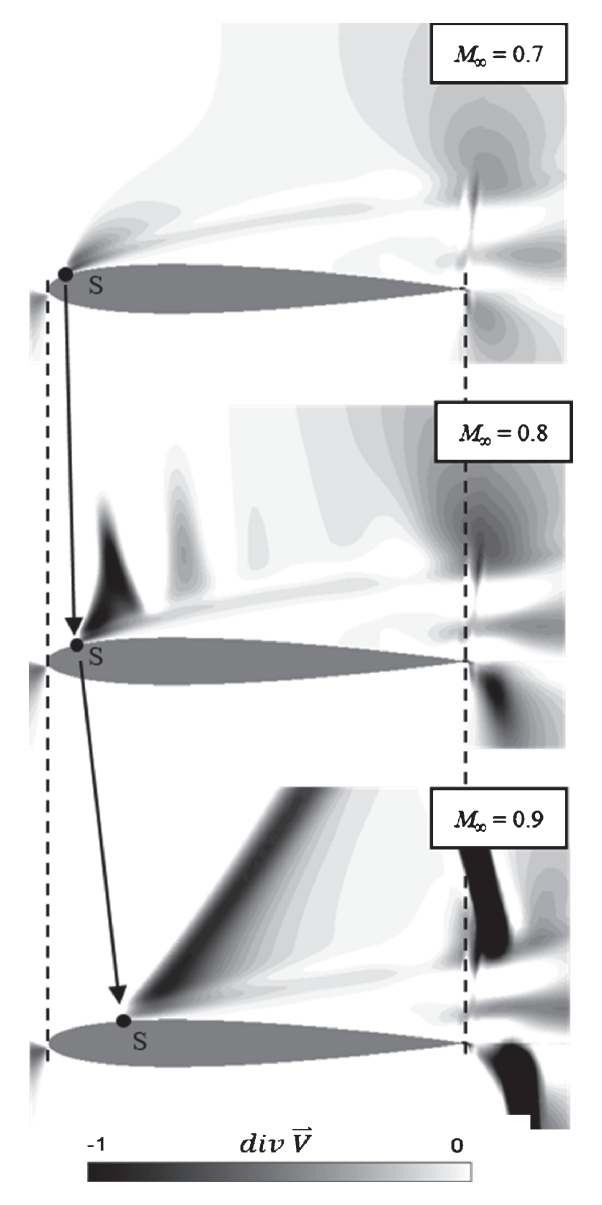

Fig. 12. Velocity-divergence distribution at $\alpha=9^{\circ}$ (S denotes start point of shock wave generation). 


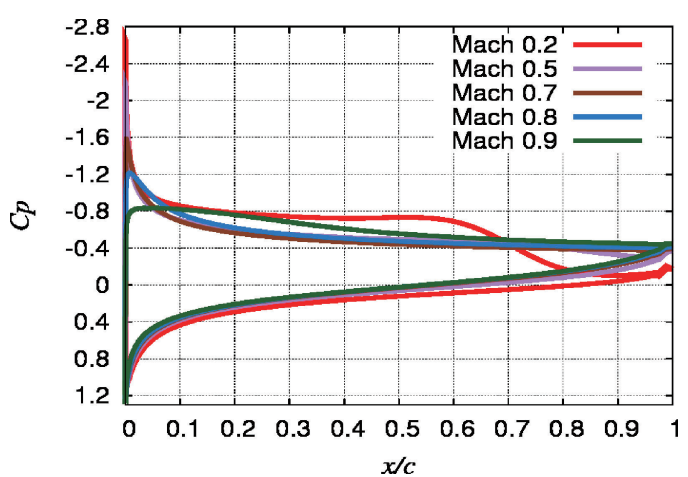

Fig. 13. $C_{p}$ distributions on the upper surface of NACA0002 at $\alpha=6^{\circ}$.

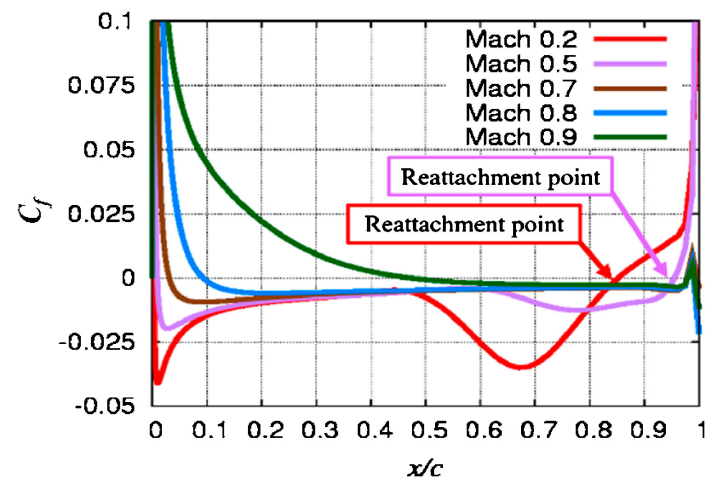

Fig. 14. $C_{f}$ distributions on the upper surface of NACA0002 at $\alpha=6^{\circ}$.

moves the downstream as the Mach number increases.

\subsubsection{Geometric characteristics of different airfoils}

To better understand the rapid decrease in $C_{l}$ for the thin airfoils in Fig. 7, we analyzed the flow around the NACA0002 airfoil at an angle of attack of $6^{\circ}$. The distributions of $C_{p}$ and $C_{f}$ are plotted in Figs. 13 and 14. The corresponding distributions of $x$-direction time-averaged flow velocity and instantaneous vorticity are shown in Figs. 15 and 16 , respectively. The $C_{p}$ distribution on the upper surface at $M_{\infty}=0.2$ remains constant from $x / c=0.20$ to 0.65 , and a marked decrease in $C_{f}$ occurs around $x / c=0.65$. Then, the recovery of $C_{f}$ is found at approximately $x / c=0.85$. Turning to the time-averaged flow field at $M_{\infty}=0.2$, as shown in Fig. 15(a), the flow separation occurs at the leading edge, and the flow is reattached on the airfoil surface. The region of the reattachment flow on the airfoil surface is located at approximately $x / c=0.85$ where the recovery of $C_{f}$ is observed as shown in Fig. 14, together with vortex shedding of the separated shear layer from the leading edge. Therefore, this reattachment of the flow contributes to the large $C_{l}$. This phenomenon is mainly reported in the low-Reynolds number regime (especially, from $10^{4}$ to $10^{5}$ ), ${ }^{26)}$ and a tendency similar to that mentioned above is also confirmed in this study. Conversely, these features disappear as the Mach number increases, and the shear layer of this separation flow is stabilized, ${ }^{27)}$ preventing reattachment of flow. Thus, the constant $C_{p}$ distribution at $M_{\infty}=0.5$ is disappeared, and the marked recovery of $C_{f}$ becomes smaller. Then, the time-averaged flow-reattachment points move downstream to the trailing

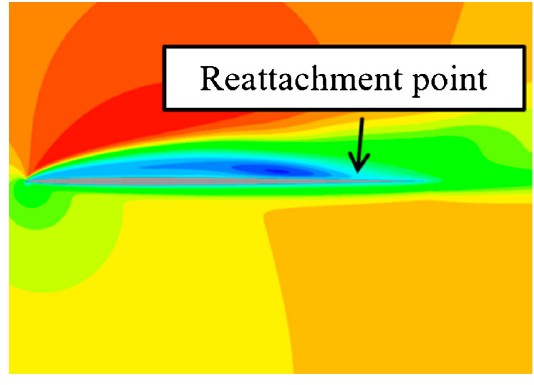

(a) $M_{\infty}=0.2$

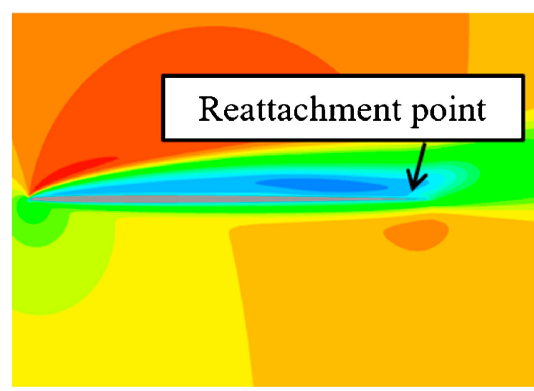

(b) $M_{\infty}=0.5$

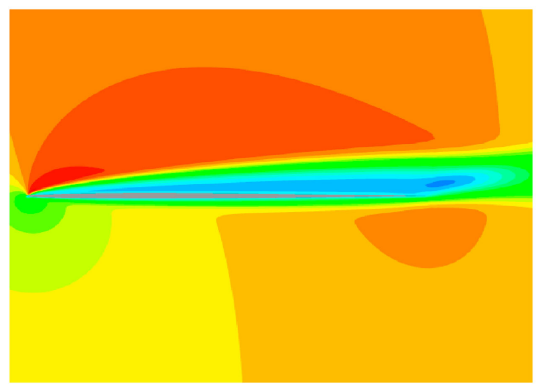

(c) $M_{\infty}=0.7$

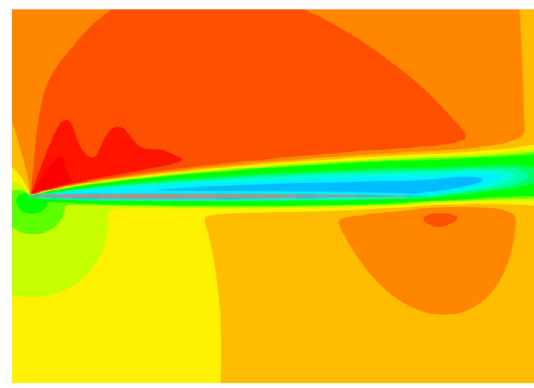

(d) $M_{\infty}=0.8$

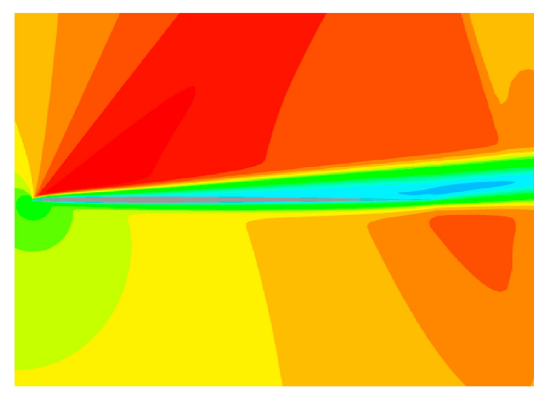

(e) $M_{\infty}=0.9$

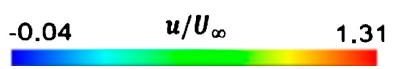

Fig. 15. Time-averaged flow fields at different Mach numbers at $\alpha=6^{\circ}$. 


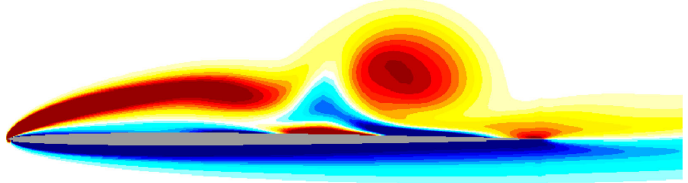

(a) $M_{\infty}=0.2$

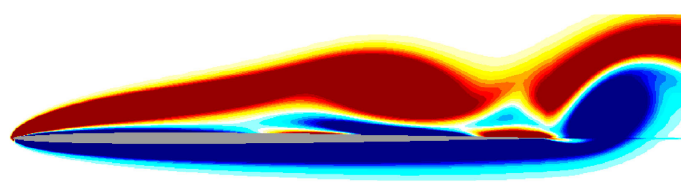

(b) $M_{\infty}=0.5$

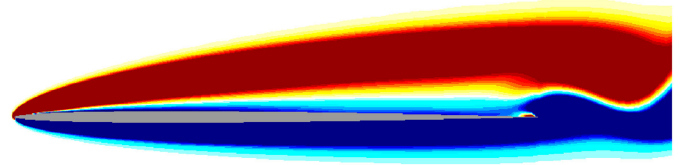

(c) $M_{\infty}=0.7$

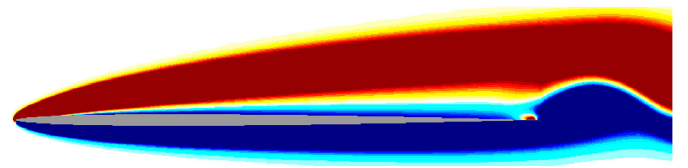

(d) $M_{\infty}=0.8$

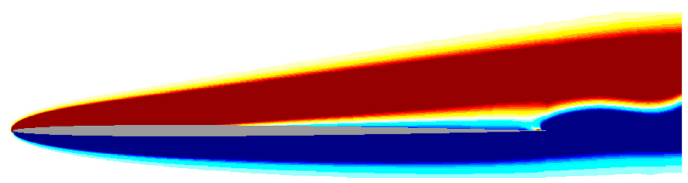

(e) $M_{\infty}=0.9$

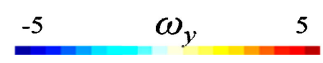

Fig. 16. Snapshots of instantaneous vorticity distributions at different Mach numbers at $\alpha=6^{\circ}$.

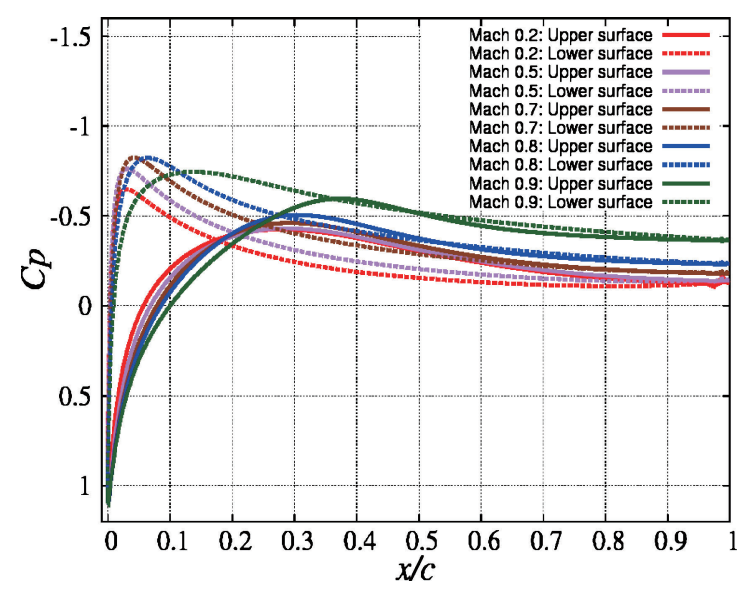

Fig. 17. $C_{p}$ distributions at NACA4412 at $\alpha=0^{\circ}$.

edge as shown in Fig. 15(b). In addition, this flow reattachment on the upper surface disappear above $M_{\infty}=0.7$. Consequently, the rapid decrease in $C_{l}$ occurs. A similar tendency is observed for the NACA4402 airfoil at $\alpha=9^{\circ}$.

Next, the effect of the Mach number on the decrease in $C_{l}$ of the NACA4412 airfoil at low angles of attack observed in Fig. 7 is discussed. The $C_{p}$ distributions of the NACA4412

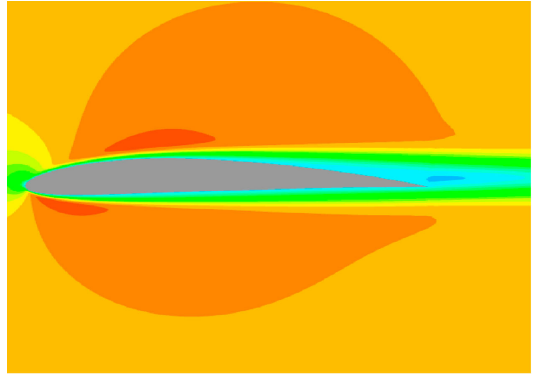

(a) $M_{\infty}=0.2$

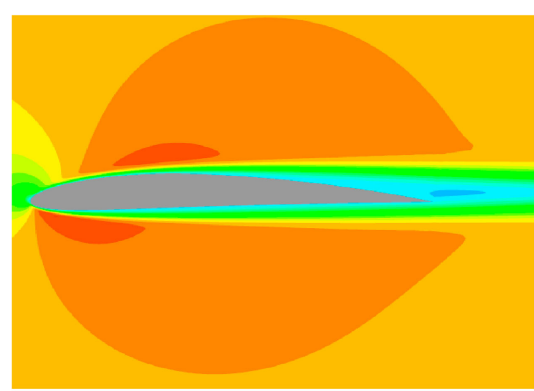

(b) $M_{\infty}=0.5$

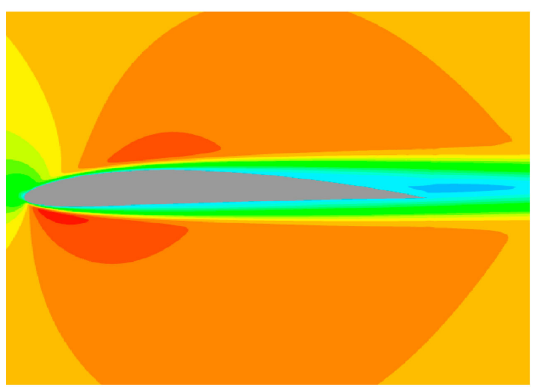

(c) $M_{\infty}=0.7$

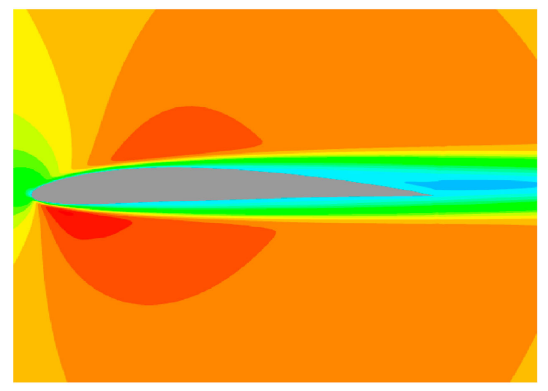

(d) $M_{\infty}=0.8$

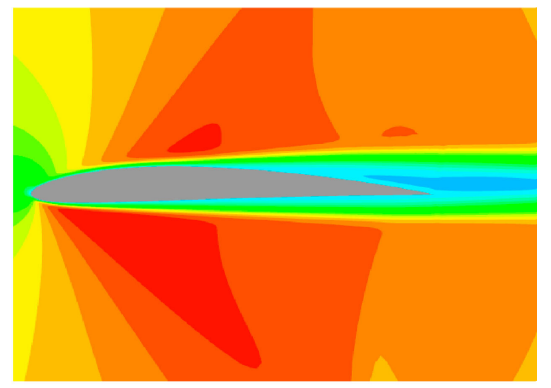

(e) $M_{\infty}=0.9$

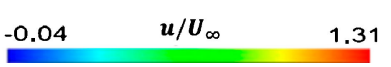

Fig. 18. Time-averaged flow fields for different Mach numbers at $\alpha=0^{\circ}$. 
airfoil at $\alpha=0^{\circ}$ are plotted in Fig. 17. The corresponding time-averaged flow velocity distributions in the $x$-direction are shown in Fig. 18. When the Mach number is small, $C_{p}$ on the lower surface is larger than that on the upper surface from approximately $x / c=0.2$ to the trailing edge, although the inequality is reversed near the leading edge. However, as the Mach number increases, $C_{p}$ on the lower surface becomes smaller, and the velocity becomes larger than that on the upper surface. The high-speed region on the lower side of the leading edge is enlarged compared with the high-speed region on airfoil's upper side, as shown in Fig. 18. In addition, as shown in Fig. 17, a clear magnitude of peak pressure at the leading edge is not observed and a rapid pressure increase does not occur. Therefore, the decreases in $C_{l}$ on cambered airfoils are more pronounced with changes in the Mach number, and the thickness and camber of NACA4412 airfoil contributes to the decrease in $C_{l}$. A similar tendency of decreasing $C_{l}$ is observed for NACA4402 at $\alpha=0^{\circ}$.

\section{Conclusion}

To understand the effects of Mach number at $R e=3,000$ with different airfoils (NACA0012, NACA0002, NACA4412, NACA4402) with thickness and camber geometries, NavierStokes simulations are conducted. The condition of the Reynolds number is corresponding to the cruising flight of the propeller blade for a Mars airplane.

The present study shows that thin and cambered airfoils have larger variations of $C_{l}$ than symmetric airfoils. As for the thin airfoils, $C_{l}$ rapidly increases at high angles of attack when the Mach number is low. This is because $C_{p}$ has a flat distribution on the upper surface, and a mark decrease in $C_{f}$ occurs and the recovery of $C_{f}$ is found along the chord direction of airfoils. Then, in the time-averaged flow field, the flow separation occurs at the leading edge, and the flow is reattached on the airfoil surface. On the other hand, the rapid increase in $C_{l}$ disappear as the Mach number increases. This result indicates that the flat distributions of $C_{p}$ and the recovery of $C_{f}$ are disappeared, and the flow reattachment in the time-averaged flow field does not occurs.

As for cambered airfoils, the decrease in $C_{l}$ becomes larger than that on the symmetric airfoils, and the negative value of $C_{l}$ on the NACA4412 airfoil is also observed when the Mach number is higher. This is because $C_{p}$ near the leading edge on the lower surface is smaller than that on the upper surface and the high-speed region on the lower side of the leading edge is enlarged compared with the high-speed region on airfoil's upper side as the Mach number increases. Then, the decreases in $C_{l}$ on cambered airfoils are more pronounced with changes in the Mach number, and the thickness and camber of NACA4412 airfoil contributes to the decrease in $C_{l}$.

Concerning to the influence of the viscosity at $R e=3,000$ with the Mach number effect, the results of the NACA0012 airfoil between Navier-Stokes simulations at $R e=3,000$ and the linearized potential theory (the Prandtl-Glauert rule) are also compared. Then, the critical Mach number at $R e=$ 3,000 tends to be larger than that predicted by linear theory, and a shock wave is generated at $R e=3,000$ in simulations of higher Mach number conditions. In addition, at lower angles of attack, the separation points at $R e=3,000$ move forward as the Mach number increases. These movements of the separation points at higher angles of attack are smaller than that at lower angle of attack. Then, the separation point at $M_{\infty}=0.9$ is located more on the trailing edge side than that at $M_{\infty}=0.8$ because of the generations of the shock wave.

\section{References}

1) Mueller, T. J.: Low Reynolds Number Vehicles, AGARD-AG-288, 1985.

2) Drela, M.: Transonic Low Reynolds Number Airfoils, J. Aircraft, 29, 6 (1992), pp. 1106-1113.

3) Hall, D. W., Tsai, K. C., Galbraith, D., and Parks, R. W.: Airplane for Mars Exploration: Conceptual Design of the Full-Scale Vehicle Propulsion System Concept, NASA/Ames Research Center, 940351099, 1997.

4) Guynn, M. D., Croom, M. A., Smith, S. C., and Parks, R. W.: Evolution of a Mars Airplane Concept for the ARES Mars Scout Mission, AIAA Paper 2003-6578, 2003

5) Tanaka, Y., Okabe, Y., Suzuki, H., Nakamura, K., Kubo, D., Tokuhiro, M., and Rinoie, K.: Conceptual Design of Mars Airplane for Geographical Exploration, Proceedings of the 36th JSASS Annual Meeting, April 2005, pp. 61-64 (in Japanese).

6) Oyama, A. and Fuji, K.: A Study on Airfoil Design for Future Mars Airplane, AIAA Paper 2006-1484, 2006.

7) Kojima, R., Nonomura, T., Oyama, A., and Fujii, K.: Large-Eddy Simulation of Low-Reynolds-Number Flow over Thick and Thin NACA Airfoils, J. Aircraft, 50 (2013), pp. 187-196.

8) Anyoji, M., Nonomura, T., Aono, H., Oyama, A., Fujii, K., Nagai, H., and Asai, K.: Computational and Experimental Analysis of a High Performance Airfoil under Low-Reynolds-Number Flow Condition, $J$. Aircraft, 51, 6 (2014), pp. 1864-1872.

9) Lee, D., Kawai, S., Nonomura, T., Anyoji, M., Aono, H., Oyama, A., Asai, K., and Fujii, K.: Mechanisms of Surface Pressure Distribution within a Laminar Separation Bubble at Different Reynolds Numbers, Phys. Fluids, 27, 2 (2015), 023602.

10) Sanuki, M.: Propeller, Togaku-sya, Tokyo, 1937, pp. 16-18 (in Japanese).

11) Takaki, R.: Aerodynamic Characteristics of NACA 4402 in Low Reynolds Number Flows, J. Jpn. Soc. Aeronaut. Space Sci., 54 (2006), pp. 367-373 (in Japanese)

12) Suwa, T., Nose, K., Numata, D., Nagai, H., and Asai, K.: Compressibility Effects on Airfoil Aerodynamics at Low Reynolds Number, AIAA Paper 2012-3029, 2012.

13) Yonezawa, K., Sunada, S., Otsuki, M., Anyoji, M., Nagai, H., Asai, K., and Okamoto, M.: Research Development of Propulsion Devices of Mars Exploration Airplane, Proceedings of the 58th Space Sciences and Technology Conference, 1B02, November 2014 (in Japanese).

14) Fujii, K. and Obayashi, S.: High-Resolution Upwind Scheme for Vortical-Flow Simulations, J. Aircraft, 26, 12 (1989), pp. 1123-1129.

15) Fujii, K., Endo, H., and Yasuhara, M.: Activities of Computational Fluid Dynamics in Japan: Compressible Flow Simulations, High Performance Computing Research and Practice in Japan, John Wiley \& Sons, New Jersey, 1990, pp. 139-161.

16) Fujii, K. and Yoshihara, H.: Navier-Stokes Benchmark Tests, Supercomputers and Their Performance in Computational Fluid Dynamics, Fujii, K. (ed.), Notes on Numerical Fluid Mechanics, Vol. 37, Vieweg+ Teubner Verlag, New Jersey, 1993, pp. 105-126.

17) Shima, E. and Jounouchi, T.: Role of CFD in Aeronautical Engineering (No. 14)_AUSM Type Upwind Schemes, Proceeding of the 14th NAL Symposium on Aircraft Computational Aerodynamics, 1997, pp. 7-12.

18) Van Leer, B.: Towards the Ultimate Conservative Difference Scheme, IV. A New Approach to Numerical Convection, J. Comput. Phys., 23 (1997), pp. 276-299. 
Trans. Japan Soc. Aero. Space Sci., Vol. 61, No. 6, 2018

19) Fujii, K.: Simple Ideas for the Accuracy and Efficiency Improvement of the Compressible Flow Simulation Methods, Proceedings of the International CFD Workshop on Supersonic Transport Design, Tokyo, March 1998, pp. 21-23.

20) Nishida, H. and Nonomura, T.: ADI-SGS Scheme on Ideal Magnetohydrodynamics, J. Comput. Phys., 228 (2009), pp. 3182-3188.

21) Anyoji, M., Numata, D., Nagai, H., and Asai, K.: Effects of Mach Number and Specific Heat Ratio on Low-Reynolds-Number Airfoil Flows, AIAA J., 53, 6 (2015), pp. 1640-1654.

22) Lee, D. H., Nonomura, T., Oyama, A., and Fujii, K.: Comparison of Numerical Methods for Evaluating Airfoil Aerodynamics Characteristics at Low-Reynolds Number, J. Aircraft, 52, 1 (2015), pp. 296-306.

23) Anderson, J. D., Jr.: Fundamentals of Aerodynamics, Fifth Edition, McGraw-Hill, New York, 2011, pp. 722-736.
24) Seling, M. S.: Low Reynolds Number Airfoil Design, von Karman Institute for Fluid Dynamics (VKL) Lecture Series, no. RTO/AVTVKT-104, 2003

25) Schlichiting, H.: Boundary-Layer Theory, Seventh Edition, McGrawHill, New York, 1979, pp. 356-358.

26) McMasters, J. H. and Henderson, M. L.: Low Speed Single Element Airfoil Synthesis, Technical Soaring, 6 (1980), pp. 1-21.

27) Sandham, N. D. and Reynolds, W. C.: Compressible Mixing Layer: Linear Theory and Direct Simulation, AIAA J., 28, 4 (1990), pp. 618-624.

Jinsoo Cho Associate Editor 